seph Calabrese 283 Patienten mit bipolaren Störungen im ambulanten Setting rekrutieren. Die Patienten erhielten alle OPT - optimized personal treatment eine auf dem texanischen Algorithmusprojekt basierende Behandlungsstrategie, bei der von PsychopharmakologieExperten anhand verschiedener Kriterien eine pharmakologische Behandlung für den Patienten empfohlen wird (vergleichbar mit einer Leitlinien-gerechten Therapie). Zusätzlich wurde die Hälfte der Patienten mit niedrig dosiertem Lithium behandelt - die Zuordnung zu den Gruppen erfolgte dabei randomisiert. Aufgrund des speziellen Studiendesigns war eine Verblindung nicht möglich. Alle Patienten wurden über sechs Monate begleitet und neben dem klinischen Schweregrad (erfasst mit der für bipolare Störungen abgewandelten CGI-Skala) wurde die Häufigkeit von Anpassungen der Pharmakotherapie als primärer Endpunkt betrachtet. Es zeigten sich keine Unterschiede zwischen den Gruppen hin- sichtlich der Hauptfragestellungen oder hinsichtlich der Remissionsraten. Deutlich mehr Teilnehmer in der LithiumGruppe beendeten die Studie vorzeitig (26\% vs. $2 \%$ ). Der einzige Vorteil in der Lithium-Gruppe war eine geringere Verschreibungshäufigkeit von Antipsychotika zusätzlich zu den „mood-stabilizers“, ein besonders interessanter Punkt, da in der Lithium-Gruppe mehr Patienten im Jahr vor Studieneinschluss unter (hypo-)manen Episoden litt als in der OPT-Gruppe.

Kommentar: Die LiTMUS-Studie ist in mehrfacher Hinsicht ein Meilenstein: Nicht nur, da die Wissenschaftler sich eines in fast allen Bereichen der Psychopharmakotherapie wenig beforschten Themas - Kombinationsund Augmentationsstrategien im klinischen Alltag - annehmen, sondern auch, da mit der Einführung des primären Endpunktes der "Medical adjustments needed" ein für alltagsnahe Studien tauglicher Parameter beschrieben wird, der gerade bei fluktuierenden Erkrankungsbildern hilfreich sein kann. Eindrucksvoll ist ferner, dass diese Studie im Rahmen eines herausragenden Netzwerks von Bipolarforschern innerhalb von zwei Jahren durchgeführt werden konnte. Dennoch sollten auch zwei wichtige Kritikpunkt genannt werden: Zum einen wurden in die Studie auch Patienten eingeschlossen, die in der Vergangenheit bereits nicht auf Lithium respondiert hatten, womit die Studie einen gewissen Bias gegen Lithium enthält. Zum anderen erreichten zahlreiche Patienten nur Lithium-Spiegel unterhalb von $0,4 \mathrm{mmol} / \mathrm{l}$, womit der therapeutische Bereiche wahrscheinlich unterschritten wurde. Es bleibt daher immer noch Forschungsbedarf zur Frage der niedrig dosierten Lithium-Augmentation bei bipolaren Störungen. Prof. Dr. Helge Frieling

Nierenberg AA et al. Lithium treatment moderate-dose use study (LiTMUS) for bipolar disorder: a randomized comparative effectiveness trial of optimized personalized treatment with and without lithium. Am J Psychiatry 2013; 170: $102-10$

\section{Ketamin bei therapieresistenter bipolarer Depression: Versuchen Sie es mal intramuskulär!}

\begin{abstract}
Die breite Verwendung von Ketamin als Antidepressivum wird, trotz guter Studienlage, durch die intravenöse Applikationsform eingeschränkt. Die orale Gabe ist aufgrund der niedrigen Bioverfügbarkeit wenig befriedigend, die intranasale Applikation schlecht steuerbar. Abhilfe könnte die intramuskuläre Gabe von Ketamin bieten.
\end{abstract}

$\mathrm{D}$ ie Gruppe von Maurizion Fava berichtet im American Journal of Psychiatry von zwei Patientinnen mit therapieresistenter Depression bei Bipolar-IIStörung, die im Rahmen von ärztlichen Heilversuchen mit Ketamin intramuskulär behandelt wurden. Bei einer Patientin konnte zuvor ein guter therapeutischer Effekt mit der I.v.-Gabe von 0,5 $\mathrm{mg} / \mathrm{kg}$ Ketamin über 40 Minuten an jedem zweiten Tag erzielt werden, dieser war nach Beendigung der Behandlung aber nicht anhaltend. Die orale und intranasale Gabe zeigten keine Effekte. Die Patientin erhielt dann alle vier Tage zunächst $32 \mathrm{mg}$, dann $50 \mathrm{mg}$ Ketamin intramuskulär, worunter sie innerhalb weniger Tage eine vollständige Remission der depressiven Symptome erlebte. Nach sechsmonatiger Behandlung kam es zu erneuten Symptomen, die durch eine Dosissteigerung auf $70 \mathrm{mg}$ alle vier Tage wiederum in Remission gebracht werden konnten. Die Patientin litt unter Reizbarkeit, Alpträumen und dissoziativen Gefühlen als Nebenwirkungen. Im zweiten Fall wurde die intramuskuläre Gabe von Ketamin nach erfolgloser orale Therapie mit $100 \mathrm{mg}$ begonnen und dann, aufgrund von ausgeprägtem dissoziativen Erleben in reduzierter Dosis $(50 \mathrm{mg})$ alle drei Tage fortgeführt. Hier kam es zu einer Verbesserung der Symptomatik innerhalb von einer Woche. Ebenfalls nach sechs Monaten kam es zu einem Rückfall, der durch die Zugabe von $\mathrm{Bu}$ - propion behandelt wurde. Die Autoren führen an, dass es bei dieser Patientin nicht zur vollständigen Remission der Depression gekommen sei, sie aber nicht mehr unter Suizidalität litt und sie wieder hätte arbeiten können.

Kommentar: Der Einsatz von Ketamin bei therapieresistenten Depressionen bleibt ein spannendes Kapitel. Die intramuskuläre Gabe könnte, wenn durch weitere Studien belegt, eine interessante Alternative zur aufwendigen I.v.-Applikation und zur oft frustranen oralen Gabe werden. Allerdings handelt es sich bislang um die ersten beiden publizierten Fälle - auf eine erste randomisierte kontrollierte Studie werden wir aber sicherlich nicht allzu lange warten müssen. Bemerkenswert an diesem als Letter to the Editor veröffentlichen Bericht ist übrigens auch, dass das "Disclosure of interest"Statement deutlich länger ist als der Artikel selbst.

Prof. Dr. Helge Frieling

Cusin C et al. Long-term maintenance with intramuscular ketamine for treatment-resistant bipolar II depression. Am J Psychiatry 2012; 169: 868-69 